\title{
Complementary DNA cloning of the pear 1-aminocyclopropane-1-carboxylic acid oxidase gene and agrobacterium-mediated anti-sense genetic transformation
}

\author{
$\mathrm{JING}^{1}{ }^{1}, \mathrm{ZHEN} \mathrm{DONG}^{2}$ and YU-XING ZHANG ${ }^{3}$ \\ ${ }^{1}$ College of Biology Science and Engineering, Hebei University of Economics and Business, \\ Shijiazhuang, Hebei 050061; ${ }^{2}$ Hebei Women's Vocational College, Shijiazhuang, Hebei 050091; \\ ${ }^{3}$ College of Horticulture, Agriculture University of Hebei, Baoding, Hebei 071001, P.R. China
}

Received October 21, 2014; Accepted July 17, 2015

DOI: $10.3892 / \mathrm{mmr} .2015 .4419$

\begin{abstract}
The aim of the present study was to genetically modify plantlets of the Chinese yali pear to reduce their expression of ripening-associated 1-aminocyclopropane-1-carboxylic acid oxidase (ACO) and therefore increase the shelf-life of the fruit. Primers were designed with selectivity for the conserved regions of published $\mathrm{ACO}$ gene sequences, and yali complementary DNA (cDNA) cloning was performed by reverse transcription quantitative polymerase chain reaction (PCR). The obtained cDNA fragment contained 831 base pairs, encoding 276 amino acid residues, and shared no less than $94 \%$ nucleotide sequence identity with other published ACO genes. The cDNA fragment was inversely inserted into a pBI121 expression vector, between the cauliflower mosaic virus 35S promoter and the nopaline synthase terminator, in order to construct the anti-sense expression vector of the ACO gene; it was transfected into cultured yali plants using Agrobacterium LBA4404. Four independent transgenic lines of pear plantlets were obtained and validated by PCR analysis. A Southern blot assay revealed that there were three transgenic lines containing a single copy of exogenous gene and one line with double copies. The present study provided germplasm resources for the cultivation of novel storage varieties of pears, therefore providing a reference for further applications of anti-sense RNA technology in the genetic improvement of pears and other fruit.
\end{abstract}

Correspondence to: Dr Jing Qi, College of Biology Science and Engineering, Hebei University of Economics and Business, 47 Xuefu Road, Shijiazhuang, Hebei 050061, P.R. China E-mail: qijing_2@126.com

Key words: yali pear, 1-aminocyclopropane-1-carboxylic acid oxidase, reverse transcription-quantitative polymerase chain reaction, antisense expression vector, genetic transformation

\section{Introduction}

The Chinese yali pear, Pyrus bretschneideri Rehd., is one of the main cultivated pear varieties in northern China and is an important fruit of export earnings. It is not only popular due to its sweet taste and nutritious value, but also due to its high water content. The medicinal values of the yali pear include re-hydration of the body, moistening of the lungs, hepatoprotective properties and relieving of cough and asthma. In recent years, with the continuous improvement of the standard of living in China, the demand for yali pears has increased. In order to provide yali pears throughout the year, it is required to delay their ripening, preserve their freshness and extend their shelf-life, which is currently a major challenge for the yali pear industry (1). Numerous studies on the extension of the storage period of yakli pears have been performed, which indicated that the effects of the external environment at harvest as well as during storage and processing affect the aging of yali pears $(2,3)$. A series of preservation measures were developed; however, these measures were not able to fundamentally control the aging of pears (4). Therefore, in order to overcome the challenge of extending the shelf-life of yali pears, it is required to generate novel varieties of yali pear by modifying their genes associated with storage-associated aging characteristics.

As the pear ripens, its respiratory rate increases, and in the climacteric stage, increased levels of ethylene, which has an important regulatory role in its ripening, are released (5). Therefore, reducing endogenous synthesis of ethylene in yali is a fundamental way to extend its storage and preservation period. The biosynthetic pathway of ethylene in plants is as follows: Methionine is converted in to $S$-adenosylmethionine, which is then transformed into 1-amino-cyclopropane-1-carboxylic acid (ACC), which is then oxidized by ACC oxidase (ACO) to form ethylene (6). As ACC is the direct precursor of ethylene, decreases in the content of ACO in plants directly affect the in vivo synthesis of ethylene (7). Based on the important regulatory role of $\mathrm{ACO}$ in the endogenous synthesis of ethylene, the aim of the present study was to inhibit the expression of ACO by anti-sense RNA technology. For this, complementary DNA (cDNA) fragments of the ACO gene and anti-sense expression 
vectors were constructed and subsequently transfected into pear plantlets using agrobacterium-mediated genetic transformation technology. Anti-sense genetic transformation of the ACO gene was performed in the pear plantlets in order to provide germplasm resources for the cultivation of novel storage varieties of pears, therefore providing a reference for further applications of anti-sense RNA technology in the genetic improvement of pears and other fruit.

\section{Materials and methods}

Materials. Fully mature Yali fruit was collected from the Park of Hebei Agricultural University on the 25th August. Ripe and disease-free fruits were picked and transported to the lab in ice boxes $\left(4^{\circ} \mathrm{C}\right)$ for cryopreservation.

The Escherichia $(E$.$) coli strain DH5a, the cloning vector$ pUCm-T, an UNIQ-10 Column Medi-Preps small-amount plasmid extraction kit (B511241) and a DNA gel extraction kit (UNIQ-10 Column MicroDNA Gel Extraction kit; B511139) were purchased from Shanghai Sangon Co. (Shanghai, China); Oligo (dT) 15, Moloney murine leukemia virus reverse transcriptase (M-MLV RTase), Taq enzyme, desoxynucleotide triphosphate (dNTP), restriction endonucleases, T4 ligase, a genome extraction kit (Takara MiniBEST Plant Genomic DNA Extraction kit; 9768) and DNA markers (1 kb DNA Ladder, DL 2000 Marker, Wide-Range DNA Ladder and 250 bp DNA Ladder) were purchased from Takara Bio Inc., (Otsu, Japan); digoxin labeling and detection kits (DIG DNA Labeling and Detection kit 11093657910) were provided by Roche Diagnostics (Basel, Switzerland); other conventional chemical reagents were all obtained from Sangon Biotech Co., Ltd. (Shanghai, China). The eukaryotic expression vector pBI121 and agrobacterium LBA4404 were provided by Dr Xiao-Li Liu (Biological Science and Engineering College, Hebei Economics University, Shijiazhuang, China) and preserved in our laboratory.

Primer design. According to the conserved sequences of ACO genes published on GenBank (http://www.ncbi.nlm. nih.gov/genbank), a pair of polymerase chain reaction (PCR) primers was designed by Oligo6.0 software (Molecular Biology Insights, Inc., Colorado Springs, CO, USA). Upstream primer, 5'-TTGTGAGAACTGGGGTTTCTTTGAG-3' and downstream primer, 5'-TCATAGCTTCAAACCTTGGTTCTTT-3'; primers were synthesized by Shanghai Sangon Co.

$R N A$ isolation and reverse transcription $(R T)-P C R$. Total RNA of yali fruit was extracted and purified according to the improved cetyltrimethylammonium bromide method (8). The purified RNA was subjected to RT with M-MLV RTase with oligo (dT) 15 as the primer. The obtained RT product was used as a template for PCR amplification; the reaction was performed in a $25-\mu 1$ mixture, which contained the cDNA template (50 ng), 10X PCR buffer (2.5 $\mu$ l), Taq enzyme (1.5 units), $30 \mathrm{ng}$ of upstream and downstream primers each, $\mathrm{Mg}^{2+}(2.0 \mathrm{mmol} / \mathrm{l})$ and $\mathrm{dNTP}(0.25 \mathrm{mmol} / \mathrm{l})$. PCR was performed in a T Gradient PCR instrument (Biometra, Göttingen, Germany). The amplification program was as follows: Pre-denaturation at $94^{\circ} \mathrm{C}$ for $7 \mathrm{~min}$, denaturation at $94^{\circ} \mathrm{C}$ for $50 \mathrm{sec}$, annealing at $52^{\circ} \mathrm{C}$ for $1 \mathrm{~min}$ and extension at $72^{\circ} \mathrm{C}$ for 1 min for a total of 35 cycles; subsequently, full extension was performed at $72^{\circ} \mathrm{C}$ for $5 \mathrm{~min}$. The PCR products were separated by $1 \%$ agarose gel electrophoresis before the target fragments were collected using a DNA gel extraction kit. The target fragments were linked with pUCm-T vector and transformed into DH5 $\alpha$ competent $E$. coli cells via the heat shock (thermal stimulation conversion) method for white-blue plaque selection. In brief, selective medium plates were prepared, the prepared competent cells were thawed on ice, then $20 \mathrm{ng}$ of plasmid DNA was added and mixed gently, prior to placing on ice for $30 \mathrm{~min}$. The cell solution was added to tubes that were then placed in a $42^{\circ} \mathrm{C}$ water bath for heat shock for $90 \mathrm{sec}$, then were rapidly transferred to a centrifuge tube in an ice bath for 1-2 min. A total of $400 \mu 1$ Lysogeny Broth medium (Sangon Biotech Co., Ltd.) was then added with gentle shaking at $37^{\circ} \mathrm{C}$ for 45 minutes to allow for the recovery of bacteria. The cells were then cultured for 12-16 h for the observation of white colonies at $37^{\circ} \mathrm{C}$. Positive recombinant colonies were selected and the plasmid was extracted by a small-amount extraction kit after amplification and reproduction; after restriction enzyme digestion (XbaI/Sacl), the bacteria were sequenced by Shanghai Sangon Co.

Reverse insertion of the pear ACO gene into the expression vector and agrobacterium transduction. The sequenced fragment of the ACO gene was inversely inserted into the multiple cloning site of the pUCm-T vector to obtain a recombinant plasmid, which was double-digested by XbaI/SacI together with eukaryotic expression vector pBI121. After electrophoretic separation, the target gene fragments and linear vector pBI121 without Gus gene were recovered. Following connecting with $1 \mu 1$ 10X ligation buffer (Sangon Biotech Co., Ltd.), $1 \mu 1$ PEG4000 (Sangon Biotech Co., Ltd.), $1 \mu \mathrm{l}$ pUCm-T vector, $3 \mu \mathrm{l}$ PCR Production (Sangon Biotech Co., Ltd.) and $1 \mu 1$ T4 ligase overnight, agrobacterium LBA4404 was transformed by the freeze-thaw method and recombinant colonies were screened on a Kan/Rif antibiotic tablet (Sangon Biotech Co., Ltd.). Following amplification and reproduction, the plasmid was extracted using the small-amount extraction kit, and identified by $\mathrm{XbaI} / \mathrm{SacI}$ digestion and PCR. PCR primers and reaction conditions were the same as mentioned above.

Genetic transformation of pear plants. Referring to the re-generation and genetic transformation system of Li et al $(9,10)$, genetic transformation of yali was performed. The pear leaf callus was pre-cultured for two days was used as a starting material for genetic transformation. It was incubated in a suspension of agrobacterium grown in agrobacterium culture medium (Sangon Biotech Co., Ltd.) with a optical density at $600 \mathrm{~nm}\left(\mathrm{OD}_{600}\right)$ of 0.8 for $10 \mathrm{~min}$; after removing excess bacteria with a filter paper, the leaf was inoculated in regeneration medium [NN69 + 6-benzylaminopurine (BA) $3.0 \mathrm{mg} / 1$ + indole-3-acetic acid (IAA) $0.5 \mathrm{mg} / \mathrm{l}$; Shanghai Shenggong Co., Ltd., Shanghai, China] and co-cultured in the dark for two days. Subsequently, $200 \mu \mathrm{g} / \mathrm{ml}$ cefotaxime sodium (Cef) was added as an anti-bacterial agent. The resistant buds were then transferred into regeneration-selective medium [NN69+ BA $3.0 \mathrm{mg} / 1+$ IAA $0.5 \mathrm{mg} / 1+$ Cef $200 \mathrm{mg} / 1+$ kanamycin (Kan) $5 \mathrm{mg} / \mathrm{l}$; Shanghai Shenggong Co., Ltd.]; when the length of 
adventitious buds was $4-6 \mathrm{~cm}, 1.5-2 \mathrm{~cm}$ fragments with buds or axillary were resected and inoculated in resistant-seedling selective medium (MS + BA $1.0 \mathrm{mg} / 1+1$-naphthaleneacetic acid $0.5 \mathrm{mg} / 1+$ Cef $200 \mathrm{mg} / 1+$ Kan $20 \mathrm{mg} / \mathrm{l}$; Shanghai Shenggong Co., Ltd.) to screen Kan-resistant regenerated seedlings.

PCR identification of transgenic pear. According to the NPT II gene sequence published in GenBank, a primer pair (upstream primer, 5'-CGTCTGTCGAGAAGTTTC-3' and downstream primer, 5'-TACTTCTACACAGCCATC-3') was designed. According to the method provided in the genome extraction kit of Takara Bio Inc., the DNA of Kan-resistant transgenic pear was isolated and amplified by PCR. $10 \mu \mathrm{l}$ PCR product was mixed with $2 \mu \mathrm{l} 6 \mathrm{X}$ loading buffer and separated on a $1 \%$ agarose gel with a voltage of $5 \mathrm{~V} / \mathrm{cm}$ for 20 min prior to development of the $1 \%$ gel in buffer solution with heating, and capturing of images with the Nikon D90 microscope (Nikon Corporation, Tokyo, Japan).

Southern blot analysis of transgenic pear. According to the $35 \mathrm{~S}$ promoter sequence, the upstream primer (5'-AGGCTTACGCAGCAGGTCTCAT-3') and downstream primer (5'-GGAAGGGTCTTGCGAAGGAATG-3') were designed. Taking the pBI121 plasmid as a template, the Southern hybridization probe was prepared by PCR amplification, and then Southern blot was hybridized with the EcoRI-digested genome of transgenetic pear. Probe preparation and Southern hybridization were performed according to the instructions of the digoxin labeling and detection kit (Roche Diagnostics) to detect copies of transferred anti-sense ACO gene in the genome.

Data repetition. All experiments were repeated a total of five times, in order to verify the results.

\section{Results}

Cloning and analysis of cDNA fragments of the pear ACO gene. According to the conserved sequence of the ACO gene, a pair of specific primers were designed; using the total RNA of a ripe pear as template, a cDNA fragment of $850 \mathrm{bp}$ was obtained by RT-PCR amplification, as shown in Fig 1 . Following being recovered and purified, the fragment was inserted into a pUCm-T vector and transformed into $E$. coli DH5 $\alpha$ competent cells; after white-blue plaque selection, positive recombinant colonies were selected and the plasmid was extracted by a small-amount extraction kit after amplification and reproduction; after double digested by EcoRI and XbaI, electrophoresis was performed; the results are shown in Fig. 2. Digestion of the recombinant plasmid provided two fragments; apart from the pUCm-T vector fragment of $2,800 \mathrm{bp}$, an inserted fragment of $\sim 850 \mathrm{bp}$ was present, which was consistent with the length of the amplified fragment, confirming that the amplified fragments had been successfully inserted into the recombinant plasmid.

Sequencing of the digested positive colonies. The sequencing results (produced by Shanghai Sangon Co.) indicated that the cloned cDNA fragment was $831 \mathrm{bp}$, the largest reading frame

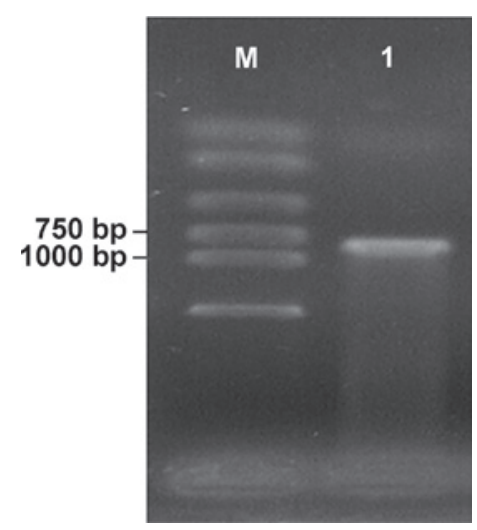

Figure 1. Agarose gel of electrophoretically purified reverse transcription quantitative polymerase chain reaction product. M, DL2000 marker; lane 1 , product.

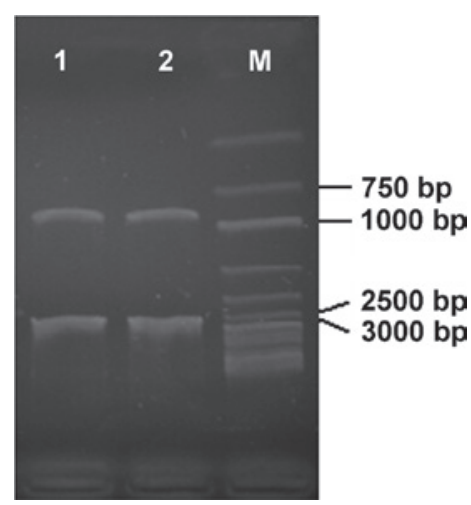

Figure 2. Electrophoresis pattern of recombinant plasmid double-digested by $E c o R I$ and $X b a I$. Lanes 1 and 2, recombinant plasmid double-digested by EcoRI and $X b a \mathrm{I} ; \mathrm{M}, 1-\mathrm{kb}$ DNA ladder.

of which was located between 2 and 829 bp with a total length of 828 bp, encoding 276 amino acid residues (Fig. 3). In total, nine cDNA sequences of pear ACO gene were retrieved from GeneBank: EF451060 (Xingshui pear), D67038 (Changshilang pear), EU047708 (snow pear), AB265797 (Yali), X87097 (Paz - Qassam pear), EU047709 (Golden Pear), EU047710 (Qieli), AJ504857 (Portugal crisp pear) and AB042111 (Changshilang pear). Using DNASTAR software, version 7.0 (DNASTAR, Inc., Madison, WI, USA), these sequences were compared with the cloned fragment (PY-ACO1) in nucleic acid sequence homology (Fig. 4); the analysis showed that the cloned gene shared no less than $94 \%$ identity with other published ACO genes of nine varieties of pear, and was $~ 100 \%$ homologous to that of the Xingshui pear, the Changshilang pear and the snow pear. This showed that the obtained fragment had a high identity with other published ACO genes, confirming that the present fragment was a cDNA fragment of the ACO gene of the Yali pear. This fragment was named as PY-ACO1 and was given the GenBank accession number EU333282.

Construction of ACO anti-sense expression vector. Two modes (positive and negative) were available for PY-ACO1 insertion into the pUCm-T vector; the sequenced plasmid with reverse insertion was selected and double-digested by 
TTGTGAGAACTGGGGTTTCTTTGAGTTGGTGAACCATGGGATATCTACTGAGCTTTTGGA $\begin{array}{llllllllllllllllllll}C & E & N & W & G & F & F & E & L & V & N & H & G & I & S & T & E & L & L & D\end{array}$ CACTGTGGAGAAGATGACCAAGGATCACTACAAGAAGACCATGGAGCAAAGGTTTAAGGA $\begin{array}{llllllllllllllllllll}T & \mathrm{~V} & \mathrm{E} & \mathrm{K} & \text { II } & \mathrm{T} & \mathrm{K} & \mathrm{D} & \mathrm{H} & \mathrm{Y} & \mathrm{K} & \mathrm{K} & \mathrm{T} & \text { M } & \mathrm{E} & \mathrm{Q} & \mathrm{R} & \mathrm{F} & \mathrm{K} & \mathrm{E}\end{array}$ AATGGTGGCAGCCAAAGGCCTCGACGCTGTCCAGTCCGAAATCCACGACTTGGACTGGGA

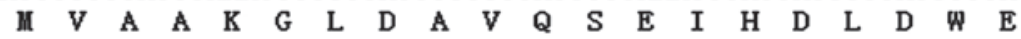
AAGCACCTTCTTCTTGCGCCACCTTCCTTCCTCAAACATTTCCGAAGTCCCTGATCTCGA

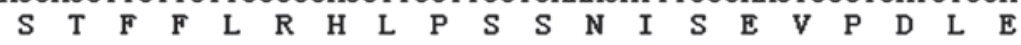
GGAAGATTACAGGAAGACCATGAAAGAATTTGCAGTGGAACTGGAGAAGCTAGCTGAGAA

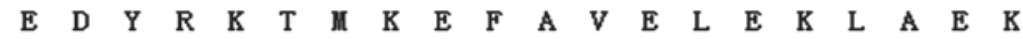
GCTTTTGGACTTGCTGTGTGAGAATCTTGGACTCGAGAAGGGTTATCTGAAGAAGGTTTT

$\begin{array}{llllllllllllllllllll}\text { L } & \text { L } & \text { D } & \text { L } & \text { L } & \text { C } & \text { E } & \text { N } & \text { L } & \text { G } & \text { L } & \text { E } & \text { K } & \text { G } & \text { Y } & \text { L } & \text { K } & \text { K } & \text { V } & \text { F }\end{array}$ CTATGGATCCAAGGGTCCGAATTTTGGGACCAAGGTCAGCAACTACCCTCCATGCCCCAA

$\begin{array}{llllllllllllllllllll}\mathbf{Y} & G & S & K & G & P & N & F & G & T & K & V & S & N & Y & P & P & C & P & K\end{array}$ GCCAGACCTGATCAAGGGACTCCGGGCCCACAGCGACGCCGGTGGCATCATCCTGCTTTT

$\begin{array}{llllllllllllllllllll}P & D & \text { L } & \text { I } & \text { K } & G & \text { L } & \text { R } & \text { A } & \text { H } & \text { S } & \text { D } & \text { A } & \text { G } & \text { G } & \text { I } & \text { I } & \text { L } & \text { L } & \text { F }\end{array}$ CCAGGATGACAAGGTCAGCGGCCTCCAGCTTCTCAAGGATGGTGAATGGGTGGATGTCCC $\begin{array}{llllllllllllllllllll}Q & \text { D } & \text { D } & \text { K } & \text { V } & \text { S } & \text { G } & \text { L } & \text { Q } & \text { L } & \text { L } & \text { K } & \text { D } & \text { G } & \text { E } & \text { W } & \text { V } & \text { D } & \text { V } & \text { P }\end{array}$ CCCAATGCACCACTCCATTGTCATAAACTTAGGTGACCAGATTGAGGTGATCACCAATGG

$\begin{array}{llllllllllllllllllll}\mathbf{P} & \text { II } & \text { H } & \text { H } & \text { S } & \text { I } & \text { V } & \text { I } & \text { N } & \text { L } & \text { G } & \text { D } & \text { Q } & \text { I } & \text { E } & \text { V } & \text { I } & \text { T } & \text { N } & \text { G }\end{array}$ GAAGTACAAGAGTGTGATGCACCGGGTGATAGCTCAGTCGGACGGGACCAGAATGTCGAT

$\begin{array}{llllllllllllllllllll}K & Y & K & S & V & \text { II } & H & R & \text { V } & \text { I } & A & Q & S & D & G & T & R & \text { II } & S & I\end{array}$ AGCCTCGTTCTACAACCCAGGCGATGATGCCTTTATCAGCCCAGCACCGGCAGTGCTTGA

$\begin{array}{llllllllllllllllllll}\text { A } & \text { S } & \text { F } & \text { Y } & \text { N } & \text { P } & \text { G } & \text { D } & \text { D } & \text { A } & \text { F } & \text { I } & \text { S } & \text { P } & \text { A } & \text { P } & \text { A } & \text { V } & \text { L } & \text { E }\end{array}$ GAAGAAAACTGAGGACGCCCCAACTTATCCCAAGTTTGTGTTTGATGACTACATGAAGCT $\begin{array}{lllllllllllllllllll}781 & \text { GTATTCTGGCCTGAAATCCAAGCCAAAGAACCAAAGTTGAAGCTATGA } \\ 261 & \text { Y } & \text { S } & \text { G } & \text { L } & \text { K } & \text { F } & \text { Q } & \text { A } & \text { K } & \text { E } & \text { P } & \text { K } & \text { F } & \text { E } & \text { A } & \text { M }\end{array}$

$\begin{array}{llllllllllllllllllll}\mathbf{K} & \mathrm{K} & \mathrm{T} & \mathrm{E} & \mathbf{D} & \mathbf{A} & \mathrm{P} & \mathrm{T} & \mathrm{Y} & \mathrm{P} & \mathrm{K} & \mathbf{F} & \mathrm{V} & \mathrm{F} & \mathbf{D} & \mathbf{D} & \mathrm{Y} & \mathbf{M} & \mathrm{K} & \mathrm{L}\end{array}$

Figure 3. Nucleotide and deduced amino acid sequences of the complementary DNA fragment encoding 1-aminocyclopropane-1-carboxylic acid oxidase from yali fruit.

\begin{tabular}{|c|c|c|c|c|c|c|c|c|c|c|c|c|}
\hline \multicolumn{12}{|c|}{ Percent identity } & \multirow[b]{3}{*}{ AB042111 } \\
\hline & 1 & 2 & 3 & 4 & 5 & 6 & 7 & 8 & 9 & 10 & & \\
\hline \multirow{11}{*}{ 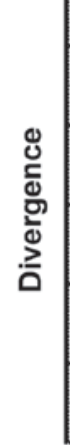 } & & 94.1 & 88.9 & 85.9 & 88.2 & 93.9 & 93.4 & 93.0 & 94.8 & 90.0 & 1 & \\
\hline & 5.9 & & 97.0 & 98.7 & 98.7 & 98.3 & 97.8 & 97.7 & 98.4 & 97.2 & 2 & AB265797 \\
\hline & 9.6 & 2.9 & & 96.2 & 97.7 & 96.8 & 96.1 & 96.4 & 97.7 & 97.7 & 3 & AJ504857 \\
\hline & 9.0 & 1.1 & 3.3 & & 96.2 & 98.7 & 98.1 & 97.7 & 99.6 & 96.5 & 4 & D67038 \\
\hline & 9.0 & 1.1 & 2.1 & 2.1 & & 98.7 & 98.1 & 97.7 & 99.6 & 98.1 & 5 & EF 451060 \\
\hline & 6.3 & 1.6 & 3.2 & 1.2 & 1.2 & & 98.6 & 98.2 & 99.3 & 97.0 & 6 & EU047708 \\
\hline & 6.8 & 2.0 & 3.7 & 1.6 & 1.6 & 1.9 & & 97.3 & 98.8 & 96.5 & 7 & EU047709 \\
\hline & 6.1 & 1.9 & 2.4 & 1.1 & 1.1 & 1.2 & 1.7 & & 97.7 & 96.7 & 8 & EU047710 \\
\hline & 5.4 & 1.4 & 2.3 & 0.4 & 0.4 & 0.7 & 1.5 & 1.1 & & 98.0 & 9 & PY-ACO1 \\
\hline & 8.4 & 2.2 & 1.9 & 3.0 & 1.6 & 2.6 & 3.1 & 1.8 & 1.7 & & 10 & X87097 \\
\hline & 1 & 2 & 3 & 4 & 5 & 6 & 7 & 8 & 9 & 10 & & \\
\hline
\end{tabular}

Figure 4. Sequence distance assay of 1-aminocyclopropane-1-carboxylic acid oxidase genes from different cultivars of pears.

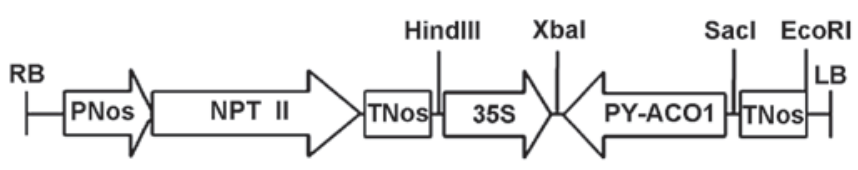

Figure 5. Diagram of the anti-sense expression vector of PY-ACO1. ACO, 1-aminocyclopropane-1-carboxylic acid oxidase; RB, T-DNA right border; PNos, Nos promotor; TNos, terminator Nos; 35S, CaMV 35S promoter; LB, T-DNA left border.

$X b a \mathrm{I} / S a c \mathrm{I}$; small pieces were recovered by electrophoresis, and directionally linked with the same digested linear vector pBI121 in order to construct the anti-sense expression vector for pear ACO. The vector map is shown in Fig. 5.

The obtained anti-sense expression vector was used to transform agrobacterium LBA4404; single colonies were selected using a Kan/Rif antibiotic tablet for propagation;

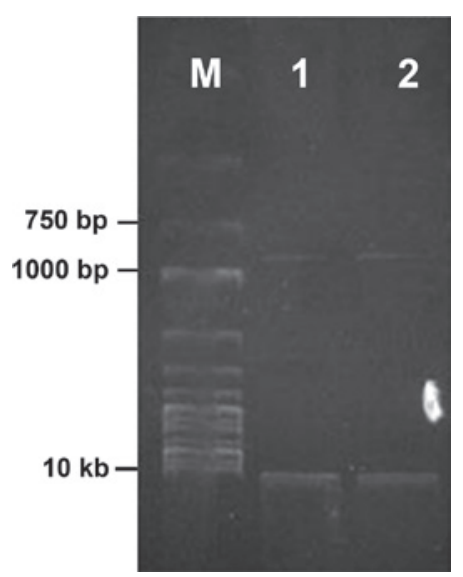

Figure 6. Electrophoretic pattern of anti-sense expression vector of PY-ACO1 double-digested by EcoRI and XbaI. M, 1-kb DNA ladder; lanes 1 and 2, anti-sense expression vector of PY-ACO1. ACO, 1-aminocyclopropane-1-carboxylic acid oxidase. 


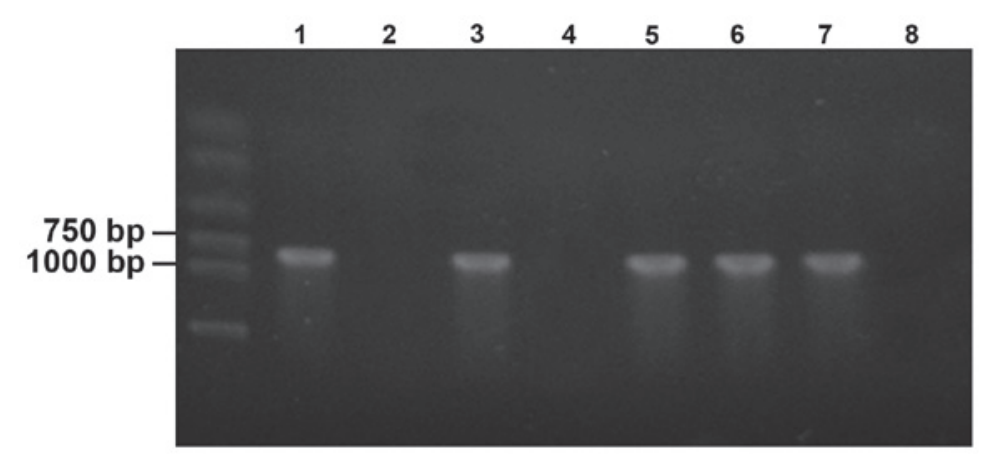

Figure 7. Polymerase chain reaction analysis of transgenic yali plant. Lane 1, positive control; lane 2, negative control; lanes 3-8, transgenic yali plants.

the recovered plasmids were double-digested by $\mathrm{XbaI} / \mathrm{SacI}$. Analysis of the products showed that digestion provided a fragment of $\sim 850 \mathrm{bp}$ (Fig. 6), consistent with the length of the PY-ACO1 fragment. The extracted plasmid was further identified by PCR using PY-ACO1 as the primer, and the amplification result is shown in Fig. 1, confirming once again that the anti-sense expression vector of pear ACO was successfully constructed and had been transformed into agrobacterium LBA4404, which were then used in studies of genetic transformation.

Transformation of yali by agrobacterium-mediated genetic anti-sense expression vector. Genetic transformation of yali was performed referring to the regeneration and genetic transformation system of Li et al (10). The pear leaf callus pre-cultured for two days was used as a starting material for genetic transformation. It was incubated in the agrobacterium suspension with an $\mathrm{OD}_{600}$ of 0.8 for $10 \mathrm{~min}$ and then inoculated in regeneration medium and co-cultured in the dark for two days; subsequently, $200 \mu \mathrm{g} / \mathrm{ml}$ cefotaxime sodium was added as an anti-bacterial agent. 412 resistant buds were obtained within a week. The resistant buds were then transferred into regeneration-selective medium; when the length of the adventitious buds was $4-6 \mathrm{~cm}, 1.5-2-\mathrm{cm}$ fragments with buds or axillary were resected and inoculated in resistant-seedling selective medium; the medium was changed once intermediately, and ultimately, a total of 29 Kan-resistant Yali seedlings were obtained, accounting for $7.03 \%$ of the total resistant buds.

PCR identification of transgenic yali. The total DNA of Kan-resistant transgenic yali was extracted; a pair of specific primers were designed based on the NPT II gene sequence published in GenBank; transgenic plants of yali were identified by PCR. The results shown in Fig. 7 indicated that there were no amplification products in the negative controls (lane 2) using DNA of non-transfected yali plants as a template, while the target fragments of $800 \mathrm{bp}$ were contained in the positive control (lane 1), in which anti-sense expression vector was used as a template, and in four transgenic plants, consistent with the design length, initially confirming that the anti-sense ACO gene had been successfully transformed in four yali plants. The other 25 plants failed to amplify the target fragment and appeared as a false positive, accounting for $86.21 \%$ of all Kan-resistant plants.

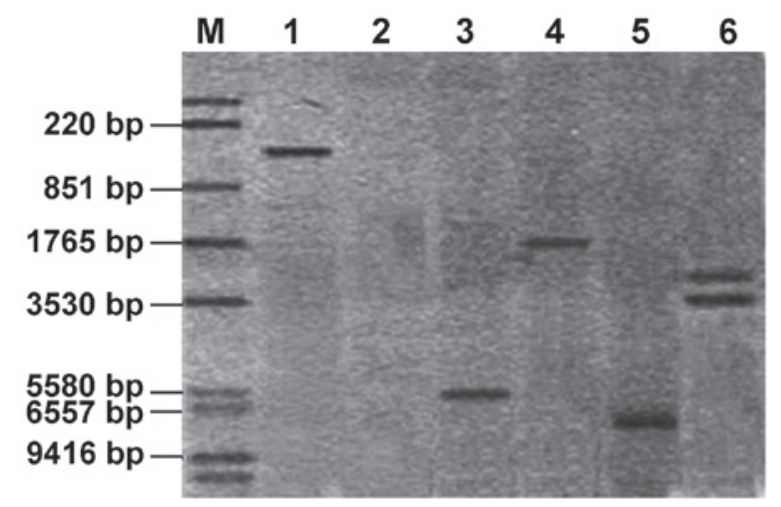

Figure 8. Southern blot analysis of transgenic yali plants. M, Dig-labeled DNA marker; lane 1, positive control; lane 2, negative control; lanes 3-6, transgenic yali plants.

Southern blot analysis of transgenic yali. The total DNA of the positive transgenic plants identified by PCR was digested with EcoRI; after electrophoresis, the fragment of the $35 \mathrm{~S}$ promoter was used as a probe to perform Southern hybridization for further identification of transgenic plants. Southern blot analysis (Fig. 8) showed that the hybridization signal was detected in four PCR-positive transgenic plants, out of which three (lanes 3-5) contained single copies and one (lane 6) contained double copies. Non-transformed control plants had no hybridization signal, confirming once again that pear ACO anti-sense gene had been successfully transformed into the four plants.

\section{Discussion}

The pear tree is a perennial fruit tree. Its long juvenile period and highly heterozygous genetic characteristics obstruct the use of traditional breeding methods. Anti-sense RNA technology provides novel methods for pear breeding with the advantage of its high specificity and short breeding cycle $(11,12)$. The present study used anti-sense RNA technology, to reversely connect the ACO gene fragment to a promoter and transfected the construct into pear plants, which greatly accelerated the pace of pear cultivation and provided possible novel varieties of pear, which are likely to display delayed aging and a longer shelf life. In anti-sense RNA technology, it is crucial to construct an expression vector containing the correct anti-sense gene fragment. In the present study, primers were designed according to 
the conserved regions of published $\mathrm{ACO}$ gene sequences, and yali cDNA was cloned using RT-PCR; the sequencing analysis showed that the cloned fragment shared high homology with ACO gene sequences of other pear varieties, which fully confirmed that the fragment was the component of the yali ACO gene. The anti-sense expression vector containing this fragment specifically inhibited the endogenous expression of the yali ACO gene. Based on the high homology of the fragment and other ACO gene sequences, the anti-sense expression vector constructed in the present study can be applied to the gene transformation of pears of any other variety, which is convenient for the application of anti-sense RNA technology in other pear varieties for extending their storage life.

Li et al (10) constructed a regeneration and genetic transformation system for yali, which was adopted in the present study. However, taking into account the different types of agrobacterium and ablastin [Li et al (10) used agrobacterium EHA105 and ablastin carbenicillin], the factors affecting the genetic transformation of yali were re-assessed in the present study (results not shown). These preliminary experiments by our group demonstrated that, apart from the concentration of agrobacterium and the infection time requiring adjustment, the other genetic transformation conditions were consistent with those of Li et al (10). Therefore, compared with the plant genotype, the impact of the agrobacterium type on the genetic transformation system was low, only differing in the incubation conditions. When applying different strains of agrobacterium on the same species for genetic transformation, the existing genetic transformation system should be appropriately adjusted according to the type of agrobacterium in order to achieve effective transformation of the exogenous gene.

In general, identification of genes on the molecular level is the most direct and simple way to assess successful transfection of plants (13). PCR amplification as well as Southern and northern blot hybridization are commonly used as molecular-level detection methods. In the present study, since the transformation of the pear plants was the insertion of the ACO anti-sense gene fragment and the plants' genome itself contained the ACO gene, the direct identification of the transferred anti-sense fragment would not have provided any useful information. Therefore, PCR amplification an Southern blot analysis of the NPT II gene and the $35 \mathrm{~S}$ promoter, which were transfected together with the anti-sense fragment, was performed in order to assess successful transfection. Although this molecular-level validation was indirect, the NPT II gene, 35S promoter and the target gene were connected in the inserted expression vector. Thus, the presence of NPT II and 35S implies the insertion of the target gene into the recipient's genome, which has been confirmed in other anti-sense genetic transformation studies (14). Due to restricted resources, northern hybridization detection of gene expression levels for the detection of the inhibitory effects of anti-sense RNA transfection in pear trees was not performed.

In transgenic studies, successfully transfected species are selected by screening with antibiotics, while the detection of false-positives is common on the molecular level $(15,16)$. In the present study, a total of 29 kanamycin-resistant seedlings were gained; however, PCR analysis identified only four strains of transformed resistant seedlings. The positive rate of PCR was $13.79 \%$. The reason for the high rate of false positives, in the present study may have been the fact that only two generations of seedlings were screened; furthermore, the concentrations of kanamycin may have been too low and the screening pressure may have been insufficient; furthermore, residual agrobacteria on the seedlings may have reduced the kanamycin concentration in the medium. The suppression of the occurrence of false-positives would save large amounts of time and effort. In order to reduce the amount of false-positive identification of seedlings, it is recommended to appropriately increase the concentration of kanamycin, tighten the screening criteria and sufficiently remove any residual agrobacteria in future experiments.

Numerous studies have confirmed that isoenzymes of ACO exist in plants. The gene coding for ACO constitutes a multi-gene family $(17,18)$. The gene family members have a range of different functions and temporal expression patterns, which affects the endogenous ethylene synthesis in the type 1 (low rate; regulation of plant organs, growth and aging) and 2 (rapid synthesis; plant reproductive organ growth regulation) climacteric phases $(6,19)$. When pear ACO anti-sense gene fragments from pear fruit were previously transfected into pear plants, ACO was expressed in the flowers and fruit only (unpublished results by our group). Therefore, it is speculated that the ACO gene is responsible for the climacteric type 2 synthesis of ethylene in the reproductive parts of the pear tree. By contrast, the generation of basal levels of ethylene in the remaining parts of the pear tree was low, while ACO was absent, indicating that the type 1 synthesis of ethylene was regulated by other ACO gene family members.

In conclusion, anti-sense genetic transformation of the ACO gene was performed in pear plantlets in order to provide germplasm resources for the cultivation of novel storage varieties of pears, therefore providing a reference for further applications of anti-sense RNA technology in the genetic improvement of pears and other fruit. In future studies, ACO should be detected in the transgenic pear species generated in the present study, which can only be performed after the flowering and fruiting of the respective trees.

\section{Acknowledgements}

The present study was supported by the Science and Technology plan of Hebei province in China (no. 11220103D-9).

\section{References}

1. Zhang H, Zhang S, Qin G, Wang L, Wu T, Qi K and Zhang S: Molecular cloning and expression analysis of a gene for sucrose transporter from pear (Pyrus bretschneideri Rehd.) fruit. Plant Physiol Biochem 73: 63-69, 2013.

2. Zhu L, Li JK, Zhang J, et al: Effects of 1-MCP treatment on Ya pear storage of different harvest. North Hortic 5: 222-224, 2009.

3. Li JZ, Yang WD, Ma J, et al: Effects of high carbon dioxide permeability film packaging on postharvest physiology and storage properties of Yali pear fruit. Storage Process 12: 16-19, 2012.

4. Liu XY, Wuyun TN and Zeng HY: Cloning, characterization and promoter analysis of S-RNase gene promoter from Chinese pear (Pyrus pyrifolia). Gene 505: 246-253, 2012. 
5. Zhang XS and Yan XX: Progress in fruit ripening by controlling ethylene. J Shandong Agric Univ 25: 487-490, 1994.

6. Yang SF and Hoffman NE: Ethylene biosynthesis and its regulation in high plants. Annu Rev Plant Physiol 35: 155-189, 1984.

7. Adams DO and Yang SF: Ethylene biosynthesis: Identification of 1-aminocyclopropane-1-carboxylic acid as an intermediate in the conversion of methionine to ethylene. Proc Natl Acad Sci USA 76: 170-174, 1979.

8. Dong Z: The study on the ACC oxidase genes clone of pear fruit (unpublished $\mathrm{PhD}$ thesis). Baoding, Agricultural University of Hebei province, 2007.

9. Li GQ, Zhang YX, Gao ZH, et al: Establishment of shoot regeneration system from leaf Yali pear. J Fruit Sci 3: 436-440, 2010.

10. Li GQ, Qi J, Gao ZH, et al: Construction and transformation for antisense expression vector of polyphenol oxidase gene in Yali pear (Pyrus bretschneideri Rehd.). J Plant Genet Resour 5 : 635-639, 2010.

11. Ding Y, Li S and Huang H: Applications of antisense-RNA technology in filamentous fungal metabolic engineering - a review. Sheng Wu Gong Cheng Xue Bao 25: 1316-1320, 2009 (In Chinese).

12. Temple SJ, Bagga S and Sengupta-Gopalan C: Down-regulation of specific members of the glutamine synthetase gene family in alfalfa by antisense RNA technology. Plant Mol Biol 37: $535-547,1998$
13. Carannante A, De Carolis E, Vacca P, Vella A, Vocale C, De Francesco MA, Cusini M, Del Re S, Dal Conte I, Cristaudo A, et al. Evaluation of matrix-assisted laser desorption ionization-time of flight mass spectrometry (MALDI-TOF MS) for identification and clustering of Neisseria gonorrhoeae. BMC Microbiol 15: 142, 2015.

14. Ye ZB: Genetic transformation with antisense cDNA of ethylene-forming enzyme and the breeding of longer shelf-life cultivar in tomato (unpublished $\mathrm{PhD}$ thesis). Huazhong Agricultural University, Wuhan, 2000.

15. Urban LA, Sherman JM, Moyer JW and Daub ME: High frequency shoot regeneration and Agrobacterium-mediated transformation of chrysanthemum (Dendranthema grandiflora). Plant Sci 98: 69-79, 1994.

16. Mitiouchkina TY and Dolgov SV: Modifieation of chrysanthemum plant and flower architecture by rolC gene from Agrobacterium rhizogenes introduction. Acta Hortic 508: 163-169, 2000.

17. Kim WT and Yang SF: Structure and expression of cDNAs encoding 1-aminocyclopropane-1-carboxylate oxidase homologs isolated from excised mung bean hypocotyls. Planta 194: 223-229, 1994.

18. Kahana A, Silberstein L, Kessler N, Goldstein RS and Perl-Treves R: Expression of ACC oxidase genes differs among sex genotypes and sex phases in cucumber. Plant Mol Biol 41: 517-528, 1999.

19. McMurchie EJ, McGlasson WB and Eaks IL: Treatment of fruit with propylene gives information about the biogenesis of ethylene. Nature 237: 235-236, 1972. 